\title{
Lifestyle and the Development of Dyslipidemia: a 4-year Follow-up Study of Middle-aged Japanese Male Office Workers
}

\author{
Noriyuki NAKANISHI ${ }^{* 1}$, Koji NAKAMURA*2, Kenji SUZUKI*3 and Kozo TATARA*1 \\ * I Department of Social and Environmental Medicine, Course of Social Medicine, Osaka Univiersiy Graduate School of Medicine, Osaka \\ *2 Medical Office, Osaka Main Office, Takenaka Corporation, Osaka \\ * 3 Japan Labor and Welfare Association, Tokyo
}

\begin{abstract}
To examine the lifestyle factors related to the development of dyslipidemia [low-density lipoprotein (LDL) cholesterol $\geq 150 \mathrm{mg} / \mathrm{dL}$, high-density lipoprotein (HDL) cholesterol $<40$ $\mathrm{mg} / \mathrm{dL}$, or triglyceride $\geq 250 \mathrm{mg} / \mathrm{dL}$ ], 979 dyslipidemia-free Japanese male office workers aged 35 to 54 years were followed up for four years. The numbers of new incidence cases during the follow-up period were 216 for high LDL cholesterol level, 109 for low HDL cholesterol level, and 78 for high triglyceride level. From the Cox proportional hazards model, adjusted hazard ratios (HRs) for the incidence of high LDL cholesterol level were 0.62 [95\% confidence interval (CI): 0.47-0.82] for consuming alcohol every day, 1.39 (95\% CI: 1.10-1.77) for 5$\mathrm{kg} / \mathrm{m}^{2}$ increase in body mass index (BMI), 1.45 (95\% CI: 1.10-1.91) for snacking between meals every day, and 1.64 (95\% CI: 1.18-2.30) for not eating vegetables every day. As for the incidence of low HDL cholesterol level, adjusted HRs for current cigarette smoking, consuming alcohol every day, and $5-\mathrm{kg} / \mathrm{m}^{2}$ increase in BMI were 1.74 (95\% CI: 1.17-2.59), 0.61 (95\% CI: $0.41-0.89$ ), and 1.79 (95\% CI: 1.29-2.46), respectively. Adjusted HRs for the incidence of high triglyceride level were 1.73 (95\% CI: 1.07-2.77) for current cigarette smoking, $1.74(95 \% \mathrm{CI}: 1.10-2.77)$ for $5-\mathrm{kg} / \mathrm{m}^{2}$ increase in BMI, and 0.50 (95\% CI: $0.27-$ $0.90)$ for working $10 \mathrm{~h}$ per day or more. These results suggest that an increase in BMI, current cigarette smoking, and snacking between meals every day is closely associated with an increased risk of atherogenic lipid profiles. On the other hand, alcohol consumption and eating vegetables every day may have an anti-atherogenic effect on serum lipid profiles.
\end{abstract}

Key words: lifestyle, incidence of dyslipidemia, follow-up study, Japanese men, middle age

\section{Introduction}

The intake of fat and cholesterol and alcohol consumption have increased remarkably with the rapid westernization of lifestyle." According to the National Nutrition Survey, serum total cholesterol levels increased by $13-16 \mathrm{mg} / \mathrm{dL}$ for both men and women between 1980 and $1990 .{ }^{2)}$ Recent increases in the levels of serum total cholesterol accompanied by changes in lifestyle warrant concern, and the relationship between lifestyle factors and serum lipid and lipoprotein levels is therefore of considerable interest.

Although effective pharmacological treatments for hyperlipidemia have been developed, long-term use of cholesterol-lowering drugs carries both costs and risks. ${ }^{3.4)}$ As the

Received Apr. 28 1999/Accepted Jul. 291999

Reprint requests to: Noriyuki NAKANISHI,

Department of Social and Environmental Medicine, Course of Social Medicine, Osaka Univiersiy Graduate School of Medicine

2-2 Yamada-oka, Suita-shi, Osaka 565-0871, Japan

TEL: +81(6)879-3911 FAX: +81(6)879-3919 majority of the burden of coronary heart disease (CHD) falls on people with only mildly to moderately elevated cholesterol levels, interventions which are economically affordable and widely applicable are needed to maximize the prevention of CHD. Furthermore, a large population could be reached by nonpharmaceutical remedies or lifestyle intervention for risk factors in which the general population may have a greater confidence regarding safety. Therefore, the eventual public health goal should be the primary prevention of lipid disorders and their sequelae.

To promote primary prevention of dyslipidemia at the workplace, the evaluation of lifestyle in the stage of primary prevention is considered important, and the lifestyle factors related to the development of dyslipidemia have to be identified. In this report based on a longitudinal population study using annual health examinations at the workplace, we have tried to identify the lifestyle factors in the chain of causation by estimating the risk of incidence of dyslipidemia among middleaged Japanese men. 


\section{Materials and Methods}

\section{Study Cohort}

To evaluate the lifestyle factors related to the development of dyslipidemia, a systematic surveillance of the incidence of dyslipidemia was conducted between 1994 and 1998 among employees of $\mathrm{T}$ Corporation, which is one of the biggest building contractors in Osaka. All Japanese male office workers aged 35 to 54 years in May 1994 were invited to attend the survey $(\mathrm{N}=1,368)$, and the participation rate was $99.9 \%(\mathrm{~N}=1,367)$. During the initial examination, fasting blood samples were drawn from an antecubital vein. Total cholesterol, high-density lipoprotein (HDL) cholesterol, and triglyceride were analyzed with Olympus AU-5000 (Olympus Japan Co, Ltd., Tokyo, Japan) by the Nihon Clinical Laboratories Inc. (Tokyo, Japan). Quality control of the laboratory was maintained internally, and the coefficients of variations were within $3 \%$ for total cholesterol, HDL cholesterol, and triglyceride during the entire survey period. Low-density lipoprotein (LDL) cholesterol was calculated with the aid of the formula of Friedewald et al.5) Subjects were classified as having dyslipidemia if their level of LDL cholesterol was $150 \mathrm{mg} / \mathrm{dL}$ or more, or HDL cholesterol was less than 40 $\mathrm{mg} / \mathrm{dL}$, or the triglyceride level was $250 \mathrm{mg} / \mathrm{dL}$ or more. The medical history and the history of use of prescribed drugs of the subjects were assessed by the examining physicians. Of the total of 1,367 subjects, $373(27.3 \%)$ were identified to be dyslipidemic at the initial examination. For 15 subjects $(1.1 \%)$ who had been undergoing medical care for dyslipidemia, the normolipidemic values were recorded. The remaining 979 subjects constituted the study cohort. Subjects who were found to have become dyslipidemic during repeat surveys up to May 1998 were defined as new incidences of dyslipidemia. Thirty-four subjects who were given anti-hyperlipidemic drugs during the observation period were also considered to be new incidence cases. Follow-up until May 1998 could be completed for only 916 subjects $(93.6 \%)$, because $63(6.4 \%)$ had transferred to another locality or had resigned or retired during the follow-up study. The numbers of incidence cases during the follow-up period were 216 for high LDL cholesterol level, 109 for low HDL cholesterol level, and 78 for high triglyceride level.

\section{Study items}

The survey at entry included a questionnaire on lifestyle and a physical examination. Data on lifestyle were obtained by interview. Selected items of lifestyle for this study were as follows: cigarette smoking, alcohol intake, overall obesity, eating breakfast, snacking between meals, considering nutritional balance, eating vegetables, coffee drinking, physical exercise, and hours of work and sleep, including the seven items identified in the studies of Belloc and Breslow ${ }^{6}$ and the two items recommended by Morimoto et al.," composed of eight items on healthy lifestyle for use in a study in Japan, based on that of Belloc and Breslow. Body mass index (BMI) was used as a measure of overall obesity. Weight and height were measured with the subjects wearing typical indoor clothing but with their shoes off. BMI was calculated as weight $/$ height $^{2}\left(\mathrm{~kg} / \mathrm{m}^{2}\right)$ and BMI of $26.0 \mathrm{~kg} / \mathrm{m}^{2}$ or more was regarded as overweight.

Data on lifestyle, except overall obesity, were treated as categorical variables: cigarette smoking (not smoking cigarettes vs smoking cigarettes currently); alcohol intake (not consuming alcohol every day vs consuming alcohol every day); eating breakfast (eating breakfast every morning vs not eating breakfast every morning); eating between meals (eating between meals every day vs not eating between meals every day); considering nutritional balance (considering nutritional balance vs not considering nutritional balance); eating vegetables (eating vegetables every day vs not eating vegetables every day); physical exercise (exercising once a week or more vs exercising hardly ever); coffee drinking (not drinking a cup of coffee every day vs drinking a cup of coffee or more every day); hours of work (working less than $10 \mathrm{~h}$ per day vs working $10 \mathrm{~h}$ per day or more); and hours of sleep (sleeping $7 \mathrm{~h}$ per night or more vs sleeping less than $7 \mathrm{~h}$ per night).

\section{Analytic procedures}

The statistical difference of lifestyle factors of the subjects at enrollment in relation to the four age groups was examined by the chi-square test. The incidence of dyslipidemia was defined in terms of the year in which dyslipidemia was identified during the serial annual health examinations. The Cox proportional hazards models were used to evaluate the univariate and multivariate relations between the characteristics at entry and the development of dyslipidemia. Risk factors for the development of dyslipidemia are dichotomous except age and BMI, and all the categorical variables were coded as 0 for no potential risk factors and 1 for potential risk factors.

Data analysis was performed with the SPSS/PC statistical package (Marija J. Norusis/ SPSS Inc., Chicago, Illinois). All reported $p$-values are two-tailed and the level of significance is $p<0.05$.

\section{Results}

Table 1 shows the characteristics of dyslipidemia-free subjects at baseline according to age. Alcohol intake, eating breakfast, physical exercise, hours of work, and hours of sleep differed significantly among the four age groups. The proportion of the subjects who drank alcohol every day was the lowest among those aged 35 to 39 years. On the other hand, the percentages of those who did not eat breakfast every morning or slept less than $7 \mathrm{~h}$ per night were the highest among those aged 35 to 39 years. The percentages of those who exercised hardly ever or worked $10 \mathrm{~h}$ per day or more decreased along with an increase in age. Cigarette smoking, overall obesity, snacking between meals, considering nutritional balance, eating vegetables, and coffee drinking did not differ significantly among the four age groups.

Table 2 shows univariate hazard ratios (HRs) for the incidence of dyslipidemia over 4 years as determined with the Cox proportional hazards model. Cigarette smoking was significantly associated with the incidence of low HDL cholesterol and high triglyceride levels. Alcohol intake had a significantly negative association with the incidence of high LDL cholesterol and low HDL cholesterol levels. BMI was significantly associated with the incidence of high LDL cholesterol, low HDL cholesterol and high triglyceride levels. Snacking between meals and eating vegetables were significantly associated with the incidence of high LDL cholesterol level Hours of work showed a significantly negative association with the incidence of high triglyceride level, and physical exercise achieved marginal statistical significance $(p<0.077)$. There were no significant associations between the incidence of dyslipidemia 
Table 1 Characteristics of dyslipidemia-free subjects at baseline

\begin{tabular}{|c|c|c|c|c|c|c|}
\hline \multirow[b]{2}{*}{ Item } & \multicolumn{5}{|c|}{ Age in Years } & \multirow[b]{2}{*}{$p$ value } \\
\hline & $\begin{array}{r}35-39 \\
(n=171) \\
\end{array}$ & $\begin{array}{r}40-44 \\
(\mathrm{n}=227) \\
\end{array}$ & $\begin{array}{r}45-49 \\
(n=348) \\
\end{array}$ & $\begin{array}{r}50-54 \\
(n=233) \\
\end{array}$ & $\begin{array}{c}\text { All ages } \\
(n=979)\end{array}$ & \\
\hline Cigarette smoking: smoking cigarettes currently & $42.7 \%$ & $48.9 \%$ & $51.1 \%$ & $45.9 \%$ & $47.9 \%$ & 0.285 \\
\hline Alcohol intake: consuming alcohol every day & $55.6 \%$ & $67.4 \%$ & $61.8 \%$ & $70.0 \%$ & $63.9 \%$ & 0.013 \\
\hline Overall obesity: $26.0 \mathrm{~kg} / \mathrm{m}^{2}$ or more in body mass index & $11.1 \%$ & $12.8 \%$ & $10.1 \%$ & $14.6 \%$ & $12.0 \%$ & 0.393 \\
\hline Breakfast habits: not eating breakfast every morning & $21.1 \%$ & $12.8 \%$ & $16.1 \%$ & $11.2 \%$ & $15.0 \%$ & 0.032 \\
\hline Snacking between meals: snacking between meals every day & $48.5 \%$ & $41.9 \%$ & $41.4 \%$ & $41.6 \%$ & $42.8 \%$ & 0.423 \\
\hline Concern with nutritional balance: not considering nutritional balance & $41.5 \%$ & $41.4 \%$ & $41.4 \%$ & $33.9 \%$ & $39.6 \%$ & 0.241 \\
\hline Vegetable consumption: not eating vegetables every day & $14.6 \%$ & $18.5 \%$ & $16.1 \%$ & $14.2 \%$ & $15.9 \%$ & 0.596 \\
\hline Coffee drinking: drinking a cup of coffee or more every day & $87.7 \%$ & $89.9 \%$ & $83.3 \%$ & $84.1 \%$ & $85.8 \%$ & 0.118 \\
\hline Physical exercise: exercising hardly ever & $53.8 \%$ & $46.3 \%$ & $46.8 \%$ & $35.2 \%$ & $45.1 \%$ & 0.002 \\
\hline Hours of work: working $10 \mathrm{~h}$ per day or more & $44.4 \%$ & $37.4 \%$ & $22.4 \%$ & $20.2 \%$ & $29.2 \%$ & $<0.001$ \\
\hline Hours of sleep: sleeping less than $7 \mathrm{~h}$ per night & $70.8 \%$ & $65.6 \%$ & $59.2 \%$ & $59.7 \%$ & $62.8 \%$ & 0.039 \\
\hline
\end{tabular}

a Chi-square test.

Table 2 Univariate hazard ratios ${ }^{\mathrm{a}}$ for the incidence of dyslipidemia over 4 years

\begin{tabular}{|c|c|c|c|c|c|c|c|c|c|}
\hline \multirow[b]{2}{*}{ Risk factor ${ }^{b}$} & \multicolumn{3}{|c|}{ High LDL cholesterol level } & \multicolumn{3}{|c|}{ Low HDL cholesterol level } & \multicolumn{3}{|c|}{ High triglyceride level } \\
\hline & $\mathrm{HR}$ & $95 \% \mathrm{CI}$ & $p$ value & HR & $95 \% \mathrm{CI}$ & $p$ value & HR & $95 \% \mathrm{CI}$ & $p$ value \\
\hline Age (5-year intervals) & 1.03 & $0.91-1.16$ & 0.685 & 1.10 & $0.92-1.31$ & 0.310 & 1.00 & $0.81-1.23$ & 0.997 \\
\hline Cigarette smoking: smo & 0.88 & $0.67-1.15$ & 0.358 & 1.59 & $1.08-2.32$ & 0.018 & 1.67 & $1.06-2.62$ & 0.027 \\
\hline Alcohol intake: & 0.61 & $0.47-0.80$ & $<0.001$ & 0.63 & $0.43-0.91$ & 0.015 & 1.06 & $0.67-1.70$ & 0.796 \\
\hline Body mass ind & 1.45 & $1.15-1.83$ & 0.002 & 1.68 & $1.23-2.29$ & $<0.001$ & 1.37 & $1.01-1.85$ & 0.045 \\
\hline Breakfast h & 1.13 & $0.79-1.61$ & 0.515 & 0.83 & $0.47-1.45$ & 0.511 & 1.12 & $0.62-2.03$ & 0.714 \\
\hline Snacking $b$ & 1.56 & $1.20-2.04$ & $<0.001$ & 1.37 & $0.94-2.00$ & 0.098 & 0.75 & $0.47-1.18$ & 0.213 \\
\hline Conce & 0.87 & 0.66 & 0.340 & 1.36 & $0.94-1.99$ & 0.106 & 0.95 & $0.60-1.49$ & 0.816 \\
\hline Vegetal & 1.50 & $1.09-2.08$ & 0.013 & 0.97 & $0.58-1.63$ & 0.904 & 1.26 & $0.71-2.20$ & 0.428 \\
\hline Coffe & 0.92 & $0.63-1.33$ & 0.639 & 1.34 & $0.73-2.43$ & 0.344 & 0.82 & $0.45-1.48$ & 0.508 \\
\hline Phy & 1.03 & $0.79-1.35$ & 0.824 & 0.92 & $0.63-1.34$ & 0.650 & 1.50 & $0.96-2.34$ & 0.077 \\
\hline $\mathrm{ng} 10 \mathrm{~h}$ per day or & 1.12 & $0.84-1.49$ & 0.438 & 1.15 & $0.77-1.72$ & 0.495 & 0.52 & $0.29-0.93$ & 0.028 \\
\hline Hours of sleep: sleeping less than $7 \mathrm{~h}$ per night & 0.96 & $0.73-1.26$ & 0.771 & 1.10 & $0.74-1.63$ & 0.629 & 1.12 & $0.70-1.79$ & 0.624 \\
\hline
\end{tabular}

${ }^{a} \mathrm{HR}$ indicates unadjusted hazard ratio; $\mathrm{Cl}$, confidence interval.

${ }^{b}$ Risk factors are dichotomous except age and body mass index.

Table 3 Multivariate hazard ratios ${ }^{\mathrm{a}}$ for the incidence of dyslipidemia over 4 years

\begin{tabular}{|c|c|c|c|c|c|c|c|c|c|}
\hline \multirow[b]{2}{*}{ Risk factor ${ }^{b}$} & \multicolumn{3}{|c|}{ High LDL cholesterol level } & \multicolumn{3}{|c|}{ Low HDL cholesterol level } & \multicolumn{3}{|c|}{ High triglyceride level } \\
\hline & $\mathrm{HR}$ & $95 \% \mathrm{CI}$ & $p$ value & HR & $95 \% \mathrm{CI}$ & $p$ value & HR & $95 \% \mathrm{CI}$ & $p$ value \\
\hline Age (5-year intervals) & 1.06 & $0.93-1.21$ & 0.381 & 1.13 & $0.94-1.36$ & 0.204 & 0.95 & $0.76-1.18$ & 0.617 \\
\hline Cigarette smoking: sm & 0.94 & $0.71-1.24$ & 0.655 & 1.74 & $1.17-2.59$ & 0.006 & 1.73 & $.07-2.77$ & 0.024 \\
\hline Alcohol intake: & 0.62 & $0.47-0.82$ & $<0.001$ & 0.61 & $0.41-0.89$ & 0.011 & 1.04 & $0.64-1.67$ & 0.881 \\
\hline Body mass in & 1.39 & $1.10-1.77$ & 0.007 & 1.79 & $1.29-2.46$ & $<0.001$ & 1.74 & $1.10-2.77$ & 0.019 \\
\hline Breakfa & 1.16 & $0.80-1.68$ & 0.437 & 0.78 & $0.44-1.39$ & 0.403 & 0.97 & -1.78 & 0.912 \\
\hline Snackin & 1.45 & $1.10-1.91$ & 0.009 & 1.16 & $0.79-1.72$ & 0.449 & 0.74 & $0.46-1.19$ & 0.216 \\
\hline Conce & 0.82 & $0.61-1.09$ & 0.167 & 1.35 & $0.92-2.00$ & 0.128 & 0.88 & 1.41 & 0.585 \\
\hline Vegetable con & 1.64 & $1.18-2.30$ & 0.004 & 0.91 & $0.54-1.55$ & 0.729 & 1.12 & $0.63-2.00$ & 0.707 \\
\hline Coffee drinking & 0.99 & $0.68-1.44$ & 0.958 & 1.25 & $0.68-2.30$ & 0.473 & 0.78 & $0.43-1.43$ & 0.425 \\
\hline Physi & 0.97 & $0.74-1.27$ & 0.817 & 0.90 & $0.61-1.33$ & 0.611 & 1.46 & $0.92-2.31$ & 0.109 \\
\hline & 1.21 & $0.89-1.63$ & 0.223 & 1.31 & $0.86-2.00$ & 0.210 & 0.50 & $0.27-0.90$ & 0.022 \\
\hline Hours of sleep: sleeping less than $7 \mathrm{~h}$ per night & 0.81 & $0.61-1.08$ & 0.156 & 1.01 & $0.67-1.53$ & 0.948 & 1.26 & $0.78-2.05$ & 0.341 \\
\hline
\end{tabular}

${ }^{2} \mathrm{HR}$ indicates adjusted hazard ratio; $\mathrm{Cl}$, confidence interval.

b Risk factors are dichotomous except age and body mass index.

and age, eating breakfast, considering nutritional balance, coffee drinking and hours of sleep.

Table 3 shows adjusted HRs for the incidence of dyslipidemia in a multivariate model in which factors independently associated with the development of dyslipidemia were identified through mutual adjustment. Factors showing a significant relationship with the incidence of high $L D L$ cholesterol were alcohol intake, BMI, snacking between meals, and eating vegetables. Adjusted HRs were 0.62 [95\% confidence interval (CI): 0.47-0.82] for consuming alcohol every day, 1.39 $(95 \% \mathrm{CI}: 1.10-1.77)$ for $5-\mathrm{kg} / \mathrm{m}^{2}$ increase in BMI, $1.45(95 \% \mathrm{CI}$ :
1.10-1.91) for snacking between meals every day, and 1.64 (95\% CI: 1.18-2.30) for not eating vegetables every day. As for low HDL cholesterol level, cigarette smoking, alcohol intake and BMI were independently associated with the incidence of low HDL cholesterol level. Adjusted HRs for cigarette smoking currently, consuming alcohol every day and $5-\mathrm{kg} / \mathrm{m}^{2}$ increase in BMI were 1.74 (95\% CI: 1.17-2.59), 0.61 (95\% CI: 0.41-0.89), and 1.79 (95\% CI: 1.29-2.46), respectively. Cigarette smoking, BMI, and hours of work showed significant relationships with the incidence of high triglyceride levels. Adjusted HRs for cigarette smoking currently, $5-\mathrm{kg} / \mathrm{m}^{2}$ increase in BMI and working $10 \mathrm{~h}$ 
per day or more were 1.73 (95\% CI: 1.07-2.77), 1.74 (95\% CI: $1.10-2.77)$, and 0.50 (95\% CI: 0.27-0.90), respectively.

\section{Discussion}

In this report on a longitudinal population study based on annual health examinations at the workplace, we have tried to identify the lifestyle factors related to dyslipidemia by estimating the risk of incidence of dyslipidemia over a 4-year period among middle-aged Japanese men. As reported in numerous previous studies, ${ }^{\text {R.12) }}$ overall obesity indicated by BMI was found to be an independent risk factor associated with increased levels of LDL cholesterol and triglyceride and reduced levels of HDL cholesterol. In this study, BMI had an independent association with the incidence of each serum lipid and lipoprotein disorder. Obesity has been found to be accompanied by an increased risk of $\mathrm{CHD},{ }^{(3)}$ but whether obesity is an independent risk factor for $\mathrm{CHD}$ is still uncertain, since $\mathrm{CHD}$ is mediated mainly through the metabolic consequences of obesity: hypertension, glucose intolerance, and diabetes mellitus as well as serum lipid and lipoprotein disorders. ${ }^{8-11},{ }^{14-16)}$ However, the fact that weight reduction in obese patients affects these other risk factors makes obesity important in the treatment of dyslipidemia. ${ }^{17}$ Although only $12.0 \%$ of our participants were deemed overweight (BMI $\geqq$ $26.0 \mathrm{~kg} / \mathrm{m}^{2}$ ), obesity should be a major target for CHD prevention in middle-aged Japanese men.

As for smoking, many studies have noted an inverse relationship between smoking and HDL cholesterol levels, 11, 12, (18-21) which in conjunction with alterations in $\mathrm{HDL}$ cholesterol Is anti-atherogenic properties is one suggested mechanism whereby smoking increases the risk of coronary arteriosclerosis. The triglyceride level in whole serum and a very low density lipoprotein (VLDL) fraction have been also found to increase with cigarette smoking. ${ }^{(9)}$ Phillips et al. ${ }^{(9)}$ have reported a positive association between the number of cigarettes smoked and beta lipoproteins and posited that this effect is secondary to the higher VLDL triglyceride levels in smokers. In this study, current cigarette smoking was independently associated with the incidence of low HDL cholesterol and high triglyceride levels. In the population surveyed for this study, half of our participants smoked cigarettes. Smoking cessation is one of the most effective factors in health education programs for middle-aged Japanese men to reduce the risk of CHD.

This study showed that daily alcohol consumption had an independently inverse association with the incidence of high LDL cholesterol and low HDL cholesterol levels. These results are consistent with the results of the Cooperative Lipoprotein Phenotyping Study and the Lipid Research Clinics Program Prevalence Study in the USA ${ }^{21,22)}$ and the randomized, controlled crossover trial in Japan. ${ }^{23)}$ It can therefore be suggested that even in a population characterized by lower serum cholesterol levels like the Japanese, alcohol has an anti-atherogenic effect by altering both $\mathrm{LDL}$ and HDL cholesterol. However, large amounts of alcohol intake are associated with higher risk of mortality from hypertension, stroke, cardiomyopathy and cirrhosis. ${ }^{24}{ }^{25)}$ Thus, a general recommendation cannot be given without reservation.

Favorable effects of regular physical activity on increased levels of HDL cholesterol and reduced levels of triglyceride have been observed. ${ }^{12.17 .2(6)}$ Nikkila et al. ${ }^{26}$ have reported that endurance training is associated with an adaptive increase in lipoprotein lipase (LPL) activity not only in skeletal muscle but also in adipose tissue and that the high HDL cholesterol levels of physically active people are probably accounted for, at least partly, by the increase in LPL activity and the concomitant rapid turnover of triglyceride-rich lipoproteins. In this study, exercising once a week or more had a univariately negative association with the incidence of low HDL cholesterol level, but did not achieve statistical significance. We assume that for our population, physical exercise may be an important preventive modality for subjects with reduced levels of HDL cholesterol, but further investigations should be needed to identify the frequency and strength of regular physical exercise to prevent reduced levels of HDL cholesterol in middle-aged Japanese men.

As for dietary habits, previous studies have linked the consumption of vegetables, fruits, and grain products rich in fibers with the reduction in serum LDL cholesterol. ${ }^{27,2 k)}$ Furthermore, several studies have shown that the addition of water-soluble fibers, such as guar gum and pectin, to the diet of healthy volunteers resulted in decreases in the level of LDL cholesterol, without any effect on the levels of HDL cholesterol or triglycerides. ${ }^{29,}{ }^{30)}$ This study showed that not eating vegetables every day was an independent risk factor for the incidence of high LDL cholesterol level. Furthermore, snacking between meals was independently related to the incidence of a high LDL cholesterol level. These results suggest that poor dietary habits have an atherogenic effects by altering serum lipid and lipoprotein profiles.

We found that hours of work had an independently negative association with the incidence of high triglyceride level. From the nutritional survey in this population, there were significant differences ( $p<0.001$, Student's t-test) among the consumed energy values for the two subgroups with regard to hours of work $[2,419 \pm 291 \mathrm{kcal} / \mathrm{day}$ for working less than $10 \mathrm{~h} / \mathrm{day}$ and $2,597 \pm 271 \mathrm{kcal} / \mathrm{day}$ for working $10 \mathrm{~h} /$ day or more (mean \pm SD)]. The negative association between triglyceride level and hours of work might be derived from the high consumption of energy related to working long hours. As for the effect of long work hours on health, working long hours has been shown to be related to coronary heart disease, stress, mental health indefinite complaints, fatigue, dissatisfaction with work, and depression. ${ }^{3(-39)}$ In this population, the number of working hours showed a close association with adaptive and maladaptive lifestyle factors, and the percentages of those who had BMI of less than $26.0 \mathrm{~kg} / \mathrm{m}^{2}$, exercised hardly ever, or slept less than $7 \mathrm{~h}$ per night were significantly higher among those who worked $10 \mathrm{~h}$ per day or more than among those who worked less than $10 \mathrm{~h}$ per day (91.3\% vs $86.7 \%, \mathrm{p}=0.047 ; 50.3 \%$ vs $43.0 \%, \mathrm{p}=0.036$; and $77.6 \%$ vs $56.7 \%, \mathrm{p}<0.001$, respectively). Further research is needed to clarify whether working long hours contributes to or is responsible for health conditions, including serum lipid and lipoprotein.

Several studies ${ }^{40.41)}$ have reported that consumption of unfiltered, boiled coffee is closely associated with raised serum cholesterol levels. The diterpenes cafestol and kahweol (nontriglyceride lipids present in coffee) are shown to be responsible for the hypercholesterolemic effects of boiled coffee. ${ }^{42)}$ As for the triglyceride level, investigations in to the effects of coffee consumption on triglycerides have variously found that it is either positively related ${ }^{43)}$ or negatively related ${ }^{197}$ to increased level of triglyceride. In this study, there was no significant association between coffee consumption and the incidence of high LDL 
cholesterol and triglyceride levels. Further investigation is needed to identify whether coffee consumed in Japan, which is mostly instant or filtered, might be a risk factor for the incidence of dyslipidemia in middle-aged Japanese men.

This study has several limitations. First, identification of incidental cases of dyslipidemia is a problem in conducting longitudinal studies of this common condition. Variability of serum lipid and lipoprotein levels makes it difficult to establish both normolipidemia at baseline and subsequent incidence of dyslipidemia in epidemiological studies in which investigators rely on a limited number of measurements of serum lipid and lipoprotein.

The second problem concerns the cases which could not be followed up until the end of observation. In this study, the follow-up rate until the end of this survey was $93.6 \%$, and nonfollowed up cases did not differ from followed up cases with respect to age and lifestyle at entry. We therefore believe that the influence of non-followed up cases on estimations of the incidence of hyperlipidemia was not very strong.

The third is that the dyslipidemia-free cohort in this study, particularly in older age groups, may not be typical of the general population. Individuals whose serum lipid and lipoprotein levels

\section{Reference}

1) Ueshima $H$. Changes in dietary habits, cardiovascular risk factors and mortality in Japan. Acta Cardiol 1990; 45: 311 27.

2) Nutrition Division, Public Health Bureau, Ministry of Health and Welfare. Kokumin Eiyo No Genjyo (The National Nutrition Survey, 1990). Tokyo: Daiichi Shuppan, 1992 (in Japanese).

3) Standing Medical Advisory Committee. Blood cholesterol testing. The cost-effectiveness of opportunistic cholesterol testing. London: Department of Health, 1990.

4) Expert Panel on Detection, Evaluation, and Treatment of High Blood Cholesterol in Adults (Grundy SM, Chairman). The second report of the National Cholesterol Education Program (NCEP) expert panel on detection, evaluation, and treatment of high blood cholesterol in adults (adult treatment panel II). Circulation 1994; 89: 1329-445.

5) Friedewald WT, Levy RI, Fredrickson DS. Estimation of the concentration of low-density lipoprotein cholesterol in plasma, without use of the preparative ultracentrifuge. Clin Chem 1972; 18: 499-502.

6) Belloc N, Breslow L. Relationship of physical health status and health practice. Preventive Medicine 1972; 1: 409-21.

7) Morimoto K, Kaneko T, Iijima K, Koizumi A. Human health situation and chromosome alterations: Sister chromatid exchange frequency in lymphocytes from passive smokers and patients with hereditary disease. In: Tice R, Hollaender A, Lambert B, Morimoto K, editors. Sister Chromatid Exchanges: Genetic Toxicology Human Studies. New York: Plenum, 1984: 501-812.

8) Grundy SM, Mok HYI, Zech L, et al. Transport of very low density lipoprotein triglycerides in varying degrees of obesity and hypertriglyceridemia. J Clin Invest 1979; 63: 1274-83.

9) Gordon T, Fisher M, Ernst N, et al. Relation of diet to LDL cholesterol, VLDL cholesterol, and plasma total cholesterol and triglycerides in white adults: The Lipid Research Clinics were already dyslipidemic when they were younger or who reported having been administered medical treatment for hyperlipidemia during the initial examination were excluded from this survey. Thus, a healthy worker effect may exist in this study.

Despite these porential limitations, our findings, obtained from a cohort of middle-aged Japanese men, support the conclusion that an increase in BMI, current cigarette smoking, and snacking between meals every day are closely associated with an increased risk of atherogenic lipid profiles. On the other hand, alcohol consumption and eating vegetables every day may have an anti-atherogenic effect by altering serum lipid profiles.

\section{Acknowledgments}

We would like to express our appreciation of all the employees and the Medical Office of the Osaka Main Office of Takenaka Corporation for their valuable cooperation for this study. We are also grateful to Ryuichi Kaneko and his colleagues at the Japan Labor and Welfare Association for collecting and coding the data accurately and consistently over a period of 4 years.

Prevalence Study. Arteriosclerosis 1982; 2: 502-12.

10) Denke MA, Sempos CT, Grundy SM. Excess body weight: an underrecognized contributor to high blood cholesterol levels in white American men. Arch Intern Med 1993; 153: 1093-103.

11) Chourdhury SR, Ueshima H, Kita Y, et al. Alcohol intake and serum lipids in a Japanese population. Int J Epidemiol $1994 ; 23: 940-7$.

12) Umeda $T$, Kono $S$, Sakurai $Y$, et al. Relationship of cigarette smoking, alcohol use, recreational exercise and obesity with serum lipid atherogenicity: a study of self-defense officials in Japan. J Epidemiol 1998; 8: 227-34

13) Hubert $\mathrm{HB}$, Feinleib $M, M c N a m a r a ~ P M$, et al. Obesity as an independent risk factor for cardiovascular disease: a 26-year follow-up of participants in the Framingham Heart Study. Circulation 1983; 67: 968-77.

14) Berchtold P, Jorgans V, Finke C, et al. Epidemiology of obesity and hypertension. Int J Obesity 1981; 5 (suppl 1): 17.

15) Hartz AJ, Rupley DC, Kalkhoff RD, et al. Relationship of obesity to diabetes: influence of obesity level and body fat distribution. Prev Med 1983; 12: 351-7.

16) Stern MP, Haffner SM. Body fat distribution and hyperinsulinemia as risk factors for diaberes and cardiovascular disease. Arteriosclerosis 1986; 6: 123-30.

17) Wood PD, Stefanick ML, Dreon DM, et al. Changes in plasma lipids and lipoproteins in overweight men during weight loss through dieting as compared with exercise. $\mathrm{N}$ Engl J Med 1988; 319: 1173-9.

18) Criqui $M H$, Wallace $R B$, Heiss $G$, et al. Cigarette smoking and plasma high-density lipoprotein cholesterol. The Lipid Research Clinics Program Prevalence Study. Circulation 1980; 62 (suppl IV): 70-6.

19) Phillips NR, Havel RJ, Kane JP. Levels and interrelationships of serum and lipoprotein cholesterol and triglycerides: 
association with adiposity and the consumption of ethanol, tobacco, and beverages containing caffeine. Arteriosclerosis $1981 ; 1: 13-24$.

20) Hegarty KM, Turgiss LE, Mulligan JJ, et al. Effects of cigarette smoking on high density lipoprotein phospholipids. Biochem Biophys Res Commun 1982; 104: 212-9.

21) Castelli WP, Doyle JT, Gordon T, et al. Alcohol and blood lipids. The Cooperative Lipoprotein Phenotyping Study. Lancet 1977; ii: 153-5.

22) Ernst N, Fisher M, Smith W, et al. The association of plasma high-density lipoprotein cholesterol with dietary intake and alcohol consumption. The Lipid Research Clinics Program Prevalence Study. Circulation 1980; 62 (suppl IV): 41-52.

23) Ueshima $H$, Mikawa $K$, Baba $S$, et al. Effect of reduced alcohol consumption on blood pressure in untreated hypertensive men. Hypertension 1993; 21 : 248-52.

24) Criqui $\mathrm{MH}$. The roles of alcohol in the epidemiology of cardiovascular diseases. Acra Med Scand 1987; 717 (suppl): 73-85.

25) Klatsky AL, Armstrong MA, Friedman GD. Risk of cardiovascular mortality in alcohol drinkers, ex-drinkers and nondrinkers. Am J Cardiol 1990; 66: 1237-42.

26) Nikkila EA, Taskinen MR, Rehunen S, Harkonen M. Lipoprotein lipase activities in adipose tissue and skeletal muscle of runners: relation to serum lipoproteins. Metabolism 1978; 27: 1661-71.

27) Jenkins DJA, Popovich DG, Kendall CWC, et al. Effect of a diet high in vegetables, fruit, and nuts on serum lipids. Metabolism 1997; 46: 530-7.

28) Jenkins DJA, Wolever TMS, Rao AV, et al. Effect on blood lipids of very high intakes of fiber in diets low in saturated fat and cholesterol. New Engl J Med 1993; 329: 21-6.

29) Haskell WL, Spiller GA, Jensen CD, et al. Role of watersoluble dietary fiber in the management of elevated plasma cholesterol in healthy subjects. Am J Cardiol 1992; 69: 4339.

30) Hunninghake DB, Miller VT, LaRosa JC, et al. Long-term treatment of hypercholesterolemia with dietary fiber. Am J Med 1994; 97: 504-8.
31) Russek HI, Zohnman BL. Relative significance of heredity, diet and occuparional stress in CHD of young adults. Am J Med Sci 1958; 235: 266-75.

32) Buell P, Breslow L. Mortality from coronary heart disease in California men who work long hours. J Chronic Dis 1960; 11: 615-26.

33) Cooper CL, Davidson MJ, Robinson P. Stress in the police service. J Occup Med 1982; 24: 30-6.

34) Richardsen AM, Burke RJ. Occupational strss and job satisfaction among physicians: sex difference. Soc Sci Med 1991; 33: 1179-87.

35) Cooper CL, Roden J. Mental health and satisfaction among tax officers. Soc Sci Med 1985; 21: 747-51.

36) McCall TB. The impact of long working hours on resident physicians. New Engl J Med 1988; 318: 775-8.

37) Ono Y, Watanabe S, Kaneko S, Matsumoto K, Miyako M. Working hours and fatigue of Japanese flight attendants. J Hum Ergol 1991; 20: 155-64.

38) Hurrel JJ, Lindström K. Comparison of job demands, control and psychosomatic complaints at different career stages of managers in Finland and the United States. Scand J Work Environ Health 1992; 18 suppl 2: 11-3.

39) Watanabe $S$, Torii J, Shinkai S, Watanabe T. Relationships between health status and working conditions and personalities among VDT workers. Environ Res 1993; 61: 258-65.

40) Aro A, Teirilä J, Gref CG. Dose-dependent effect on serum cholesterol and apoprotein B concentrations by consumption of boiled, non-filtered coffee. Atherosclerosis 1990; 83: 257 61.

41) Bak AAA, Grobbee DE. The effect on serum cholesterol levels of coffee brewed by filtering or boiling. $N$ Engl J Med 1989; 321: 1432-7.

42) Urgert R, Van der Weg G, Kosmeijer-Schuil TG, et al. Levels of the cholesterol-elevating diterpenes cafestol and kahweol in various coffee brews. J Agric Food Chem 1995; 43: 2167-72.

43) Williams PT, Wood PD, Vranizan KM, et al. Coffee intake and elevated cholesterol and apolipoproten B levels in men. JAMA 1985; 253: 1407-11. 